\title{
Identification and Characterization of a Human Prostate Cancer Specific Long Non-Coding RNA
}

\author{
Joseph Mazar and Ranjan J. Perera
}

\begin{abstract}
The human genome is transcribed in both (sense and anti-sense) directions and this dynamic process generates a large number of transcripts. Interestingly, the majority of these transcripts do not code for proteins, and among these non-protein-coding transcripts, exist a group of long non-coding RNAs (IncRNAs) known to play an important role in disease genesis and development in human. Here we report the identification and characterization of one such IncRNA specific to prostate cancer. Microarray and qRT-PCR analysis confirmed that IncRNA $A F 086453$ is consistently down-regulated in all prostate cancer cell lines surveyed in this study compared to normal prostate epithelial cells. Bioinformatics analysis revealed that this IncRNA is located immediately downstream of Forkhead Box Protein N1 (FOXN1). FOXN1 is also down-regulated in prostate cancer; therefore, $A F 086453$ may likely to be regulated by the FOXN1 regulatory units. Prostate cancer cell line PC3 was engineered to ectopically express $A F 086453$ and the transfected cells demonstrate defects in cell growth and invasion. These results suggest that $A F 086453$ may play an important role in prostate cancer development and may act as a useful diagnostic marker for prostate cancer detection in humans.
\end{abstract}

Index Terms-Gene expression, invasion, long non-coding RNAs, prostate cancer.

\section{INTRODUCTION}

The role of signaling molecules and transcription factors in cancer development is well appreciated. More recently, non-coding RNAs (microRNAs and long non-coding RNAs) have emerged as important regulatory molecules for the normal function of most eukaryotic cells. Some of these non-coding RNAs may influence cancer development because of their function as tumor suppressors or oncogenes. Long non-coding RNAs (lncRNAs) are transcribed and expressed in a developmentally and disease-regulated manner and their function in the genome is a source of great interest [1]-[7]. They are also known to control various aspects of transcriptional and post-transcriptional mRNA processing, and may further regulate mRNA function by binding and masking key elements within the coding sequence. Examples are elements that may be required for the

Manuscript received July 30, 2012; revised November 22, 2012. This work was supported in part by the North Florida Ovarian Cancer Alliance and the Florida Hospital Foundation.

The authors are with Sanford-Burnham Medical Research Institute, Orlando, FL 32827 USA (e-mail: jmazar@sanfordburnham.org, rperera@sanfordburnham.org). binding of trans-acting factors in post-transcriptional gene expression, such as splicing, transport, translation and degradation of pre-mRNA [8]. Non-coding RNAs are also transcribed by mechanisms similar to those involved in the transcription of most protein-coding genes, and therefore, not surprisingly, abnormal regulation of non-coding RNA genes may induce prostate cancer development and progression in humans. Fundamental questions about the interaction of microRNAs, mRNAs and long non-coding RNAs with prostate cancer development remain unanswered. Systematic sequencing of protein-coding genes has led to the identification of novel mutated genes involved in several cancer types [9]-[13]. We have recently demonstrated that siRNA-mediated knockdown of the melanoma up-regulated IncRNA SPRY4-ITI causes defects in cell growth and differentiation, and increases the apoptotic rate of melanoma cells [14]. However, little is known about the molecular function of this long non-coding RNA.

The primary focus of this manuscript is the characterization of one specific lncRNA, (Genbank accession ID AF086453), which was recently identified in our laboratory as one of the most highly down-regulated lncRNAs in numerous prostate cancer cell lines compared to prostate epithelial cells. This IncRNA is located immediately downstream of the transcription factor FOXN1 [15] and its regulation may be directly effected by FOXN1. We hypothesize that together; FOXN1 and lncRNA AF086453 may play a key role in prostate cancer development and progression in men.

Prostate Cancer is one of the leading causes of cancer deaths among American men. In 2010, an estimated 190,000 new cases of prostate cancer were diagnosed, and for 27,000 men the disease will likely be fatal. This observation suggests that there is an unmet need to develop better therapeutics and accurate biomarkers for prostate cancer. Our preliminary results show that ectopic expression of AF086453 in prostate cancer cells causes significant defects in both cell growth and invasion. These preliminary data, together with the increasing recognition that lncRNAs play important roles in cell transformation has led us to examine AF086453 to determine if it may be of use in future diagnostic \& therapeutic applications.

\section{MATERIALS AND METHODS}

\section{A. Cell Lines}

Cell lines were purchased from the American Type Culture Collection (Manassas, VA). Experimental studies illustrated in this manuscript used the human prostate cancer cell lines PPC-1 (stage D2), 22RW1, Du145 (stage 4), LNCaP (stage 4), and PC3 (stage 4). Prostate cancer cells were cultured at 
$37^{\circ} \mathrm{C}\left[5 \%\left(\mathrm{v} / \mathrm{v} \mathrm{CO}_{2}\right)\right]$ in vitro using RPMI 1640 media supplemented with $10 \%(\mathrm{v} / \mathrm{v})$ fetal bovine serum.

\section{B. NCode IncRNA Array}

One microgram of total RNA was labeled and hybridized to NCode human microarrays (Life Technologies ${ }^{\mathrm{TM}}$, Carlsbad, CA, USA) according to the manufacturer's protocols (www.Lifetechologies.com). An Agilent $2 \mu \mathrm{m}$ scanner was used to scan the slides and the data was normalized and analyzed using GeneSpring software (www.agilent.com). The NCode human array contains over 10,000 putative lncRNAs (>200 nt) including most of the known lncRNAs in human. Lack of coding potential was estimated by a previously described algorithm [14] that scores various characteristics of protein-coding genes, including open reading frame length, synonymous/non-synonymous base substitution rates and similarity to known protein. These arrays are the first generation of tools designed to investigate the dynamic expression of a large subset of lncRNAs in human to identify candidate genes for more detailed functional analysis. In addition to the lncRNA content, probes targeting mRNA content from RefSeq are also included, allowing discovery of coordinated expression with associated protein-coding genes.

\section{Quantitative Real-Time PCR}

Total RNA was acquired using the Trizol method (Invitrogen/LifeTechnologies) with quantitation and integrity of the sample performed using an Agilent 2100 Bioanalyzer (AgilentTechnologies, Santa Clara, CA, USA). Human prostate epithelial cell total RNA was acquired from ScienCell (ScienCell Research Laboratories). From these samples, total RNA (100 ng) was then reverse transcribed with a High Capacity cDNA kit (Applied Biosystems/Life Technologies) and qRT-PCR was carried out using SYBR Green mRNA Assays on a 7500 Real-Time PCR System (Applied Biosytems/Life Technologies) according to the manufacturer's protocols. SYBR Green primers included AF086453 qPCR For - ggaagcacaagggataccettggc \& AF086453 qPCR Rev - ggccaacctgtggaagttcttgtcac and FOXN1 qPCR For - gccattgttccacagccgg \& FOXN1 qPCR Rev - ggccaacctgtggaagttcttgtcac. SDS1.2.3 software (Applied Biosystems/Life Technologies) was utilized for comparative $\mathrm{Ct}$ analysis with GAPDH or b-actin acting as the endogenous controls.

\section{Construction of a Plasmid Expressing lncRNA AF086453}

Oligonucleotides complimentary to the IncRNA AF086453 genomic sequences were constructed (AF0806453 clone For taagcttcacatagtttaggtcetggcttaactctac \& AF0806453 clone Rev - actcgaggagaggggccagaaatgaaagcttg), containing HindIII and XhoI sites on their respective 5' and 3' ends, then amplified from human prostate epithelial cell genomic DNA (ScieCell Research Laboratories) using Amplitaq Gold (Applied Biosystems/Life Technologies). The product (620 bp) was then gel purified from a $1 \%$ Agarose gel (QIAquick gel extraction kit, Qiagen Corp.) and TOPO cloned into pCR4-TOPO (Invitrogen/Life Technologies). The vector construct was sequenced and the lncRNA AF086453 fragment was sub-cloned into pcDNA6/V5-HisA (Invitrogen/Life Technologies) using the HindIII and XhoI sites to create pcDNA6/AF086453.

\section{E. Growth Rate Assays for Transient Expression of AF086453 in PC3 Cells}

PC3 cells were grown to log phase, trypsinized, counted using an automated cell counter (Countess, Invitrogen/Life Technologies), and then seeded into wells of a 6-well plate at $10^{5}$ cells per well (in triplicate per time point per sample). Cells were then transfected with 5 ug of either pcDNA6/V5-HisA (Vector Control) or pcDNA6/AF086453 using Fugene 6 (Roche), or no plasmid at all (cells only). Media was changed after 4 hours, and cells were harvested at 24, 48, \& 72 hours to be counted (samples included: untransfected cells, "vector control" \& and expression vector transfected cells).

\section{F. Invasion Assays}

BD BioCoat growth factor reduced insert plates (Matrigel Invasion Chamber 24 well plates) were prepared by hydrating the matrix coating of the inserts with $0.5 \mathrm{mLs}$ of RPMI 1640 media (no serum) for $2 \mathrm{hrs}$ at $37^{\circ} \mathrm{C} / 5 \% \mathrm{CO}_{2}$ [16]. The hydration solution was carefully removed from the inserts and $0.75 \mathrm{mLs}$ RPMI 1640 media containing chemoattractant (10\% FBS) was added to the wells of the plate with $0.5 \mathrm{mLs}$ of cell suspension containing 30000 cells (either transfected with pcDNA6/AF086453 or pcDNA6/V5-HisA, or no plasmid) in serum-free medium added to each insert well. Invasion assay plates were incubated for $48 \mathrm{hrs}$ at $37^{\circ} \mathrm{C} / 5 \% \mathrm{CO}_{2}$. Following incubation, the non-invading cells were removed by scrubbing out the upper surface of the insert. The cells on the lower surface were stained with crystal violet, and then the trans-well membranes were mounted onto a microscope slide for visualization and analysis. All slides were scanned utilizing the Scanscope digital slide scanner, with the number of cells migrating counted using Aperio software. All data were expressed as percent invasion through the membrane relative to migration through the control membranes:

$\%$ invasion = Mean number of cells invading through the Matrigel membrane/Mean number of PC3 (wild type) cells migrating through membrane.

\section{RESUlTS AND DISCUSSION}

\section{A. Identification of a Long Non-Coding RNA Differentially Regulated in Prostate Cancer Cells}

Total RNA was isolated from prostate epithelial cells as well as the prostate cancer cell line PC3 and expression of IncRNAs were analyzed by using a non-coding microarray (NCode) to identify differentially regulated lncRNAs. NCode human microarrays contain probes to target 12,784 lncRNAs and 25,409 mRNAs. In total, we identified 22 lncRNAs that were significantly differentially expressed $(\mathrm{P}<0.015)$ in PC3 prostate cancer cells relative to prostate epithelial cells. Individual lncRNAs were further screened by qRT-PCR to confirm microarray results. Next, these differentially regulated ncRNAs were tested in a panel of five commonly used prostate cancer cell lines (PPC-1, DU145, 22RW1, LNCap and PC-3) to identify consistent trends of mis-regulation. We found that one lncRNA (Genbank accession ID AF086453) was highly down-regulated in all five prostate cancer cell lines compared to epithelial cells 
(with four of the five cell lines yielding less than $10 \%$ of the expression seen in epithelial cells). Fig. 1 depicts the relative gene expression levels of AF086453 in all five prostate cancer cells lines compared to prostate epithelial cells.

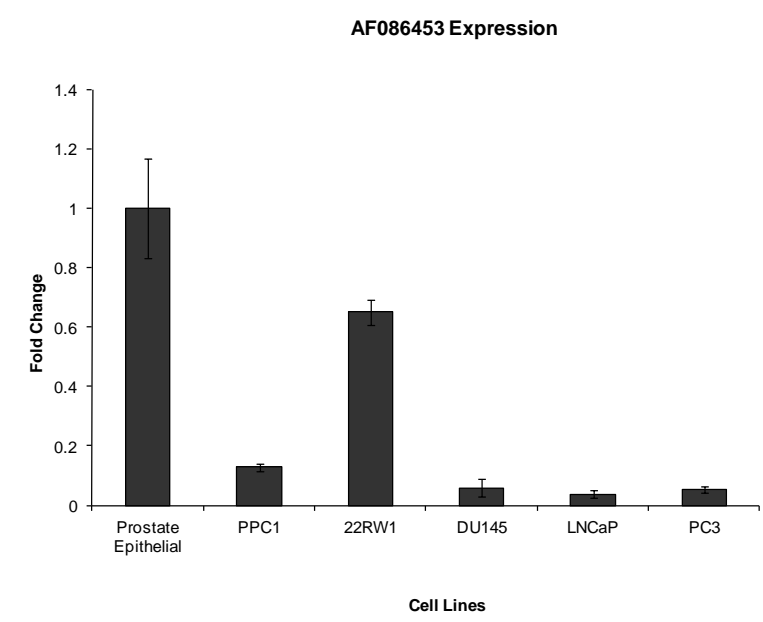

Fig. 1. AF086453 expression profiles of the prostate cancer cells lines PPC-1, 22RW1, DU145, LNCaP, and PC3 normalized to prostate epithelial cells.

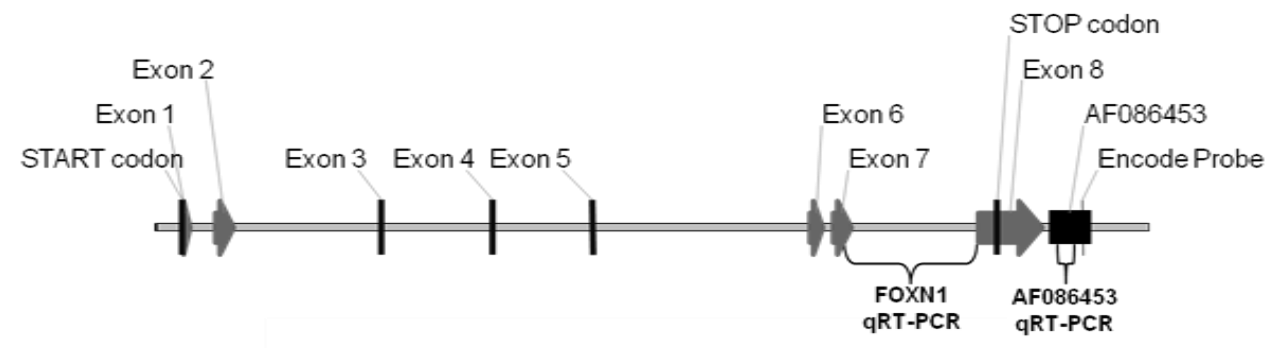

Genomic loci of AF086453 \& FOXN1 (Chromosome 17)

Fig. 2. Genomic loci of FOXN1 and lncRNA AF086453. Annotations include the locations selected for qRT-PCR in reference to FOXN1 exons and the EnCode probe initially used to measure AF086453 expression.

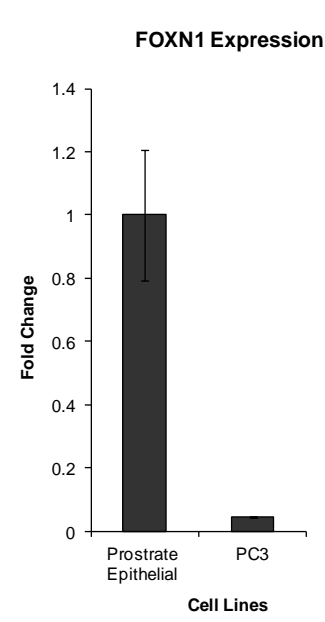

Fig. 3. qRT-PCR of FOXN1 expression in PC-3 prostate cancer cells compared to prostate epithelial cells. Experiment was performed in triplicate.

In order to determine a possible regulatory association between FOXN1 and AF086453, we performed qRT-PCR for FOXN1 in PC-3 prostate cancer cells and compared the results to prostate epithelial cells (Fig. 3). The results demonstrate that FOXN1 is highly down-regulated ( 20-fold) in prostate cancer cells. These results lead us to believe that AF086453 may be under FOXN1 regulatory control, but we are gathering additional supportive evidence in our

\section{B. FOXN1 May Regulate the Gene Expression of AF086453}

An examination of the genomic loci of AF086453 revealed that it is located less than $300 \mathrm{bp}$ downstream of the final exon of the FOXN1 gene (Fig. 2). FOXN1 is a winged-helix transcription factor that has been shown to induce terminal differentiation in keratinocytes and defects have led to T-cell immunodeficiency in the thymus [17], [18]. A study by Karanam et al. [15] indicated that FOXN1 binding sites were found to be significantly enriched in genes that were over-expressed in prostate cancer. regulation/function when a lncRNA appears within the loci or in close proximity to an Open Reading Frame [19], [20]. Our own studies involving the melanoma up-regulated lncRNA SPRY4-IT1 have supported such an observation for a possible co-regulation with the SPROUTY 4 (SPRY4) gene [14].
Previous studies have suggested coordinated

laboratory.

\section{Biological Function and the Physiological Relevance of AF086453 Down-Regulation in Prostate Cancer Cells.}

Although the down-regulation of AF086453 is clear, the physiological relevance of this lncRNA in prostate cancer cells has not been determined. In order to address this, the genomic sequence of $A F 086453$ was cloned into a eukaryotic gene expression plasmid and the plasmid was transiently transfected into prostate cancer cells (PC-3). A growth curve was performed to measure the effect of the ectopic expression of AF086453 and compared to an "empty vector" (Fig. 4A). The results indicated that after 72 hours, transfection of PC-3 cells with the AF086453-expressing plasmid (pcDNA6/AF086453) grew $~ 45 \%$ slower than either wild type or "empty vector" (pcDNA6/V5-HisA). Next, an invasion assay was performed to measure the effect of the ectopic expression of AF086453 on cell mobility. PC-3 cells were again transfected with the same construct and compared to "empty vector" or untransfected cells. All cell samples were introduced into modified Boyden invasion chambers and membranes were harvested after 48 hours. The results indicated that cells transfected with the AF086453-expressing plasmid migrated nearly 30\% slower than vector-only cells. Together, these results indicate that AF086453 expression has an adverse effect on both the growth and invasive properties of prostate cancer cells. 


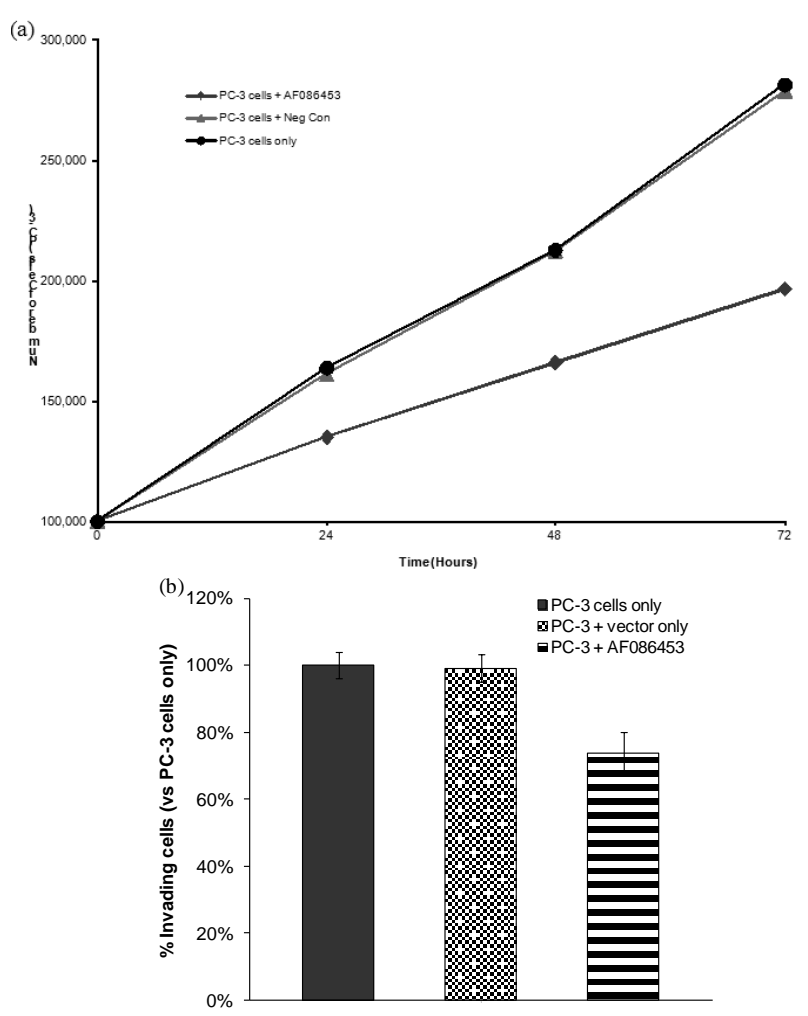

Fig. 4. The effect of ectopic expression of the IncRNA AF086453 on growth and invasion in PC-3 prostate cancer cells. A) Growth curve of PC-3 cells transfected with a plasmid expressing AF086453 or an "empty vector" plasmid versus untransfected cells. The growth curve was performed over 72 hours in triplicate. B) Invasion assay of PC-3 cells transfected with a plasmid expressing AF086453 or an "empty vector" plasmid versus untransfected cells. The invasion assay was performed over 24 hours in triplicate.

\section{CONCLUSION}

Prostate cancer represents one of the leading causes of cancer death in American men, yet the success rate of diagnosis suggests an urgent need for novel and more comprehensive approaches for future diagnostic and prognostic evaluation.

The study of long non-coding RNAs (lncRNAs) represents a new avenue of research for prostate cancer biology and may lead to the discovery of novel biomarkers and therapeutics. Our previous evaluation of non-coding RNAs in normal skin and the skin cancer melanoma offered new appraisals on the mechanisms of cellular regulation and mis-regulation associated with cancer and it is our belief that this model can be applied to the study of prostate cancer.

The results of non-coding RNA (NCode) microarrays revealed that numerous IncRNAs were differentially expressed in prostate cancer cells. One particular IncRNA, Genbank accession ID AF086453, was highly down-regulated in a panel of prostate cancer cell lines compared to prostate epithelial cells. Located immediately upstream to $A F 086453$ is the transcription factor FOXN1, known to regulate numerous cancer specific genes. Gene expression analysis confirmed that FOXNI is also down-regulated in prostate cancer which suggests a coordinated gene expression between these two genes. It is our hypothesis that AF086453 may influence FOXN1 regulation and together FOXN1 and AF086453 may play key roles in prostate cancer development in men.

Preliminary cell biology studies to examine the physiological ramifications of the ectopic expression of
AF086453 in prostate cancer cells revealed defects in both cell growth and invasion. This suggests a possible role of $A F 086453$ as a tumor suppressor either alone or in conjunction with FOXN1. Together, these results strongly justify the need for future detailed examinations of AF086453 and FOXN1 molecular functions in prostate cancer development in humans and continues to be an ongoing study in our laboratory.

\section{REFERENCES}

[1] C. Esau, X. Kang, E. Peralta et al., "Microrna-143 regulates adipocyte differentiation," J Biol Chem, vol. 279, no. 50, pp. 52361-52365, 2004.

[2] J. Mazar, K. DeYoung, D. Khaitan, E. Meister, A. Almodovar, J. Goydos, A. Ray, and R. J. Perera, "The regulation of miRNA-211 expression and its role in melanoma cell invasiveness," PLoS One, vol.5, no. 11, pp. e13779, 2010.

[3] J. Mazar, S. Sinha, M. E. Dinger, J. S. Mattick, and R. J. Perera, "Protein-coding and non-coding gene expression analysis in differentiating human keratinocytes using a three-dimensional epidermal equivalent," Mol Genet Genomics, vol. 284, no. 1, pp. 1-9, 2010.

[4] R. J. Perera, S. Koo, C. F. Bennett, N. M. Dean, N. Gupta, J. Z. Qin, and B. J. Nickoloff, "Defining the transcriptome of accelerated and replicatively senescent keratinocytes reveals links to differentiation, interferon signaling, and notch related pathways," J Cell Biochem, vol. 98, no. 2, pp. 394-408, 2006.

[5] R. J. Perera and A. Ray, "Micrornas in the search for understanding human diseases," BioDrugs, vol. 21, no. 2, pp. 97-104, 2007.

[6] Y. Sun, S. Koo, N. White, E. Peralta, C. Esau, N. M. Dean, and R. J. Perera, "Development of a micro-array to detect human and mouse micrornas and characterization of expression in human organs," Nucleic Acids Res, vol. 32, no. 22, pp. e188, 2004.

[7] Y. M. Tay, W. L. Tam, Y. S. Ang et al., "MicroRNA-134 modulates the differentiation of mouse embryonic stem cells, where it causes post-transcriptional attenuation of Nanog and LRH1," Stem Cells, vol. 26, no. 1, pp. 17-29, 2008.

[8] J. Mazar, D. DeBlasio, S. S. Govindarajan, S. Zhang, and R. J. Perera, "Epigenetic regulation of microRNA-375 and its role in melanoma development in humans," FEBS Lett, vol. 585, no. 15, pp. 2467-2476, 2011.

[9] A. Bardelli, D. W. Parsons, N. Silliman et al., "Mutational analysis of the tyrosine kinome in colorectal cancers," Science, vol. 300, no. 5621, pp. 949, 2003.

[10] C. Greenman, P. Stephens, R. Smith et al., "Patterns of somatic mutation in human cancer genomes," Nature, vol. 446, no. 7132, pp. 153-158, 2007.

[11] D. W. Parsons, S. Jones, X. Zhang et al., "An integrated genomic analysis of human glioblastoma multiforme," Science, vol. 321, no. 5897, pp. 1807-1812, 2008.

[12] T. Sjoblom, S. Jones, L. D. Wood et al., "The consensus coding sequences of human breast and colorectal cancers," Science, vol. 314, no. 5797, pp. 268-274, 2006.

[13] P. Tan, "Divide and conquer: Progress in the molecular stratification of cancer," Yonsei Med J, vol. 50, no. 4, pp. 464-473, 2009.

[14] D. Khaitan, M. E. Dinger, J. Mazar, J. Crawford, M. A. Smith, J. S. Mattick, and R. J. Perera, "The melanoma-upregulated long noncoding RNA SPRY4-IT1 modulates apoptosis and invasion," Cancer Res, vol. 71, no. 11, pp. 3852-3862, 2011.

[15] S. Karanam and C. S. Moreno, "Confac: Automated application of comparative genomic promoter analysis to DNA microarray datasets," Nucleic Acids Res, vol. 32, no. Web Server issue, pp. W475-484, 2004.

[16] H. K. Kleinman and K. Jacob, "Invasion assays," Curr Protoc Cell Biol, chapter 12, pp. Unit 12 12, 2001.

[17] S. M. Janes, T. A. Ofstad, D. H. Campbell, F. M. Watt, and D. M. Prowse, "Transient activation of FOXN1 in keratinocytes induces a transcriptional programme that promotes terminal differentiation: Contrasting roles of FOXN1 and Akt," J Cell Sci, vol. 117, no. Pt 18, pp. 4157-4168, 2004.

[18] C. Pignata, A. Fusco, and S. Amorosi, "Human clinical phenotype associated with FOXN1 mutations," Adv Exp Med Biol, vol. 665, pp. 195-206, 2009.

[19] U. A. Orom, T. Derrien, M. Beringer et al., "Long noncoding RNAs with enhancer-like function in human cells," Cell, vol.143, no. 1, pp. 46-58, 2010.

[20] U. A. Orom, T. Derrien, R. Guigo, and R. Shiekhattar, "Long noncoding RNAs as enhancers of gene expression," Cold Spring Harb Symp Quant Biol, vol. 75, no., pp. 325-331, 2010. 